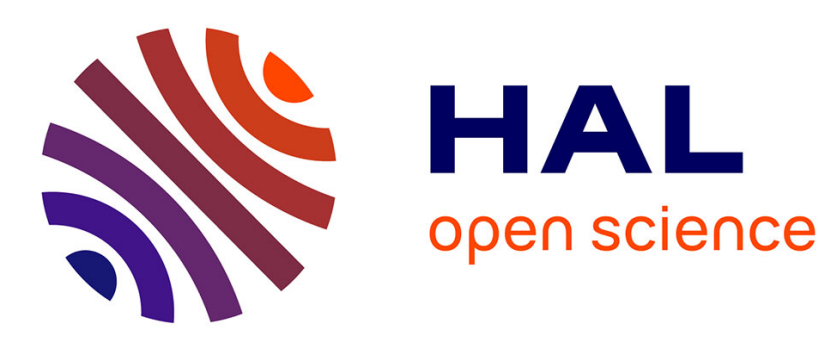

\title{
Polarization selectivity for pulsed fields in a reverberation chamber
}

Andrea Cozza, Houmam Moussa

\section{To cite this version:}

Andrea Cozza, Houmam Moussa. Polarization selectivity for pulsed fields in a reverberation chamber. 2010 Asia-Pacific Symposium on Electromagnetic Compatibility (APEMC), Apr 2010, China. pp.574577. hal-00505986

\author{
HAL Id: hal-00505986 \\ https://hal.science/hal-00505986
}

Submitted on 30 Oct 2010

HAL is a multi-disciplinary open access archive for the deposit and dissemination of scientific research documents, whether they are published or not. The documents may come from teaching and research institutions in France or abroad, or from public or private research centers.
L'archive ouverte pluridisciplinaire HAL, est destinée au dépôt et à la diffusion de documents scientifiques de niveau recherche, publiés ou non, émanant des établissements d'enseignement et de recherche français ou étrangers, des laboratoires publics ou privés. 


\title{
Polarization Selectivity for Pulsed Fields in a Reverberation Chamber
}

\author{
Andrea Cozza ${ }^{1}$, Houmam Moussa ${ }^{2}$ \\ Département de Recherche en Électromagnétisme, SUPELEC \\ 3 rue Joliot-Curie, 91192 Gif-sur-Yvette, France \\ ${ }^{1}$ andrea.cozza@supelec.fr \\ ${ }^{2}$ houmam.moussaelss. supelec.fr
}

\begin{abstract}
Time-reversal techniques have been demonstrated to be able to reverse the incoherence that usually characterizes the field distribution within reverberation chambers. Its ability of generating coherent pulsed fields has previously been applied to communications as well as the generation of controlled wavefronts for immunity testing. Nevertheless, the issue of polarization has not yet received due attention. We prove in this paper that time-reversal techniques, when applied to reverberating media, also allow controlling the polarization of the focused pulsed fields. This result, although apparently paradoxical, relies on the very incoherence properties of reverberation chambers. Remarkably, this allows to generate fields with any polarization by using one single static excitation antenna, enabling a changing polarization just by modifying the signal driving it. A theoretical model is proposed for proving this feature, together with experimental results validating it. Cross-polarization rejections much higher than $20 \mathrm{~dB}$ are observed, making the quality of the generated pulses comparable to those obtained in anechoic chambers. Interestingly, the cross-polarization rejection is not expected to depend on that of the transmission antenna as measured in a freespace environment, but just on the properties of reverberation chambers and the frequency bandwidth of the pulse.
\end{abstract}

\section{INTRODUCTION}

Reverberation chambers (RCs) are today widely used for testing how an equipment under test (EUT) reacts to an electromagnetic aggression that can be regarded as an incoherent field, constituted of a multitude of plane waves propagating along random directions with random polarizations [1]. This way of using RCs is based on narrow-band excitations, mainly in continuous-wave mode, and allows generating highintensity electric fields with a relatively low-power source. This property also makes RCs an important tool when testing non-linear responses, which may occur whenever electronic devices are exposed to high-intensity fields, and could be particularly interesting for testing high-power microwaves (HPM) effects [2]. Unfortunately in this case, it is not simply the level of the field that counts, but also the time-evolution of the aggression [3]; this is somewhat incompatible with the physics of reverberation chambers as they are currently used. Indeed, when applying pulse-like signals to the antenna exciting the $\mathrm{RC}$, the time-evolution of the field is strongly distorted, due to the highly-reverberating environment that produces a long tail of echoes following the originally intended pulse. This is indeed a strong limitation of RCs for HPM testing when

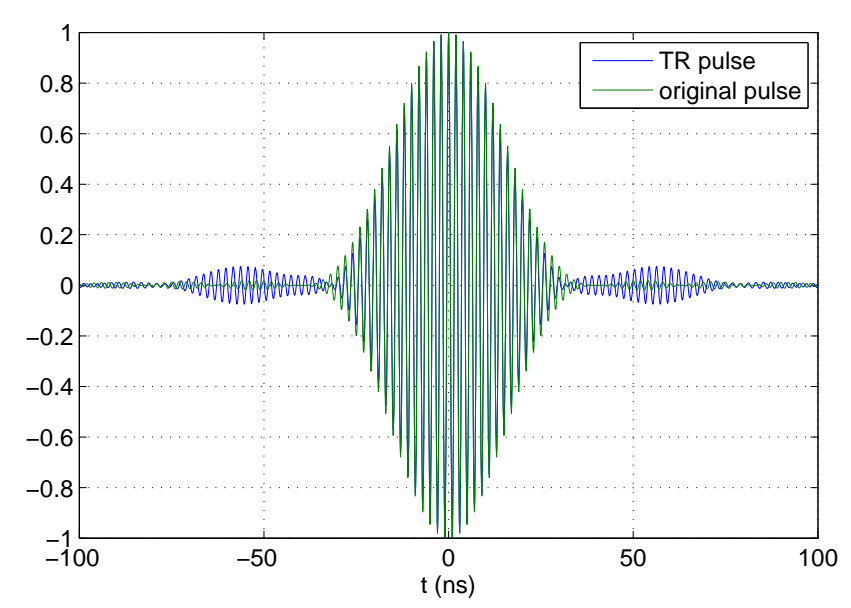

Fig. 1. An example of the ability of time-reversal techniques to generate coherent pulses in a reverberating environment. The blue curve represents the pulse received at a given location in a RC when applying time-reversal techniques, as computed by means of an experimentally measured transfer function. The green curve is the original pulse to be transmitted. The two peak-normalized curves are indistinguishable around the peak region.

compared to facilities based on pulsed radiators [4].

Nevertheless, it has been shown in [5], [6] that this trend can be reversed by means of time-reversal techniques. Pulsed fields can be transmitted within the $\mathrm{RC}$, focusing energy around a spot, about the size of the average wavelength excited, and with a time-dependency that is strongly coherent with the original pulse: an example is given in Fig. 1, as obtained from the experimental results presented in Section III. This feature is interesting since it allows creating conditions somewhat similar to those experienced in an anechoic environment, while keeping the advantages of high power efficiency typical of low-loss reverberating systems. This technique has recently been used for generating extended coherent wavefronts in RCs [7]. The main limitation of these studies is that none has yet addressed how to control the polarization of the pulsed field. This is indeed the motivation of this paper, where we prove that the incoherence properties of RCs that are the very source of its limitations in time-domain applications, can be exploited for generating coherently polarized fields thanks to time-reversal techniques.

The interest of the results here shown is not just limited 
to the ability of having a coherent polarization, but also that it can be modified without any need of mechanically steering the excitation antenna, nor to use a multitude of differently polarized antennas. Indeed, we show that the decorrelation of the field components existing in RCs, allows changing the polarization of pulsed fields just by changing the signal driving the antenna. Moreover, the cross-polarization rejection of the field can attain levels much higher than those of the same antenna used in a free-space environment. Therefore, the proposed procedure does not rely on the use of expensive antennas with a high cross-polarization rejection, but it rather makes low-cost antennas capable of generating fields with a low cross-polarization.

\section{ASYMPTOTIC CROSS-POLARIZATION PROPERTIES}

The setup investigated here is the same used in standard RC applications, i.e., a transmitting antenna placed within the $\mathrm{RC}$ in order to excite a field distribution. The vector electric field $\mathbf{E}(f, \mathbf{r})$ measured at any point $\mathbf{r}$ inside the $\mathrm{RC}$ can then be related to the power-wave $X(f)$ propagating toward the input port of the excitation antenna by means of

$$
\mathbf{E}(f, \mathbf{r})=X(f) \boldsymbol{\Phi}(f, \mathbf{r})=X(f)\left(\begin{array}{c}
\Phi_{x} \\
\Phi_{y} \\
\Phi_{z}
\end{array}\right)(f, \mathbf{r})
$$

where $\boldsymbol{\Phi}(f, \mathbf{r})$ is a vector transfer function. The three scalar transfer functions related to each Cartesian polarization component will be referred to as $\Phi_{i}(f, \mathbf{r})$ with $i=1, \ldots, 3$ for, respectively, the $x, y$ and $z$ components.

It is known that for an overmoded $\mathrm{RC}$, the $\Phi_{i}(f, \mathbf{r})$ transfer functions are submitted to the following orthogonality condition [1]:

$$
\mathrm{E}\left[\Phi_{i}(f, \mathbf{r}) \Phi_{j}^{\star}(f, \mathbf{r})\right]=C \delta_{i j},
$$

where $\mathrm{E}[\cdot]$ is the expected value operator and $C$ is a normalization constant. This condition is satisfied only when averaging over the entire space of the random realizations of the transfer functions, e.g., such as when applying mode-stirring techniques. It is worthwhile noticing that this condition has been demonstrated for an harmonic excitation. By recalling the modal theory underpinning the resonant phenomena occurring in an RC, a generic scalar transfer function can be expressed as

$$
\Phi(f)=\sum_{i=1}^{M} \gamma_{i} \psi_{i}(f)
$$

where $\psi_{i}(f)$ is the frequency response of the $i$-th resonant mode supported by the RC, centered around the frequency $f_{i}$, while $\gamma_{i} \in \mathbb{C}$ is a modal weight modelling how it is excited and received at the position $\mathbf{r}$. Equation (3) is defined over a bandwidth $B_{T}$, where the RC supports $M$ modes.

The average introduced in (2) thus applies to two distinct, though related, phenomena, usually modelled as independent processes, i.e., the modal weights $\left\{\gamma_{i}\right\}$, and the frequencies of resonance $\left\{f_{i}\right\}$ of the modes. Let us now assume that these two random processes are ergodic, i.e., that the average ensemble operator can be approximated through the arithmetic mean as applied to the different modes defining a transfer function, since ergodicity would imply [8]

$$
\lim _{M \rightarrow \infty} \mathrm{P}\left(\left|\mathrm{E}\left[\gamma_{i}\right]-\frac{1}{M} \sum_{i=1}^{M} \gamma_{i}\right|>\epsilon\right)=0 .
$$

where $\epsilon \in \mathbb{R}^{+}$. In other words, the arithmetic mean converges in probability to the ensemble average.

Assuming that the $\psi_{i}(f)$ are frequency-shifted replica of the same function, and thus that the quality factor $Q$ grows linearly over a bandwidth $B_{T}$, it follows that, although improperly stated,

$$
\lim _{M \rightarrow \infty} \int_{B_{T}} \Phi(f) \mathrm{d} f=M \mathrm{E}\left[\gamma_{i}\right] \int_{B_{T}} \psi_{i}(f) \mathrm{d} f \quad,
$$

where the integrals are computed over a bandwidth $B_{T}$ centered around the central frequency $f_{0}$. We have assumed, as often done, that the $\left\{\gamma_{i}\right\}$ are independent and identically distributed random variables as well as the $\left\{f_{i}\right\}$. Equation (5) thus implies that the ensemble average can also be approximated through an integration over the frequency variable. As long as the ergodicity assumption holds, the approach used for deriving (5) can be applied to any function of the transfer functions $\Phi_{i}(f)$. In this case, for a given realization, the mean would be applied in frequency rather than in probability. Recalling (2) and the ergodic assumption (4), the law of large numbers would thus imply that

$$
\lim _{M \rightarrow \infty} \int_{B_{T}} \Phi_{i}(f) \Phi_{j}^{\star}(f) \mathrm{d} f=M \mathrm{E}\left[\Phi_{i}\left(f_{0}\right) \Phi_{j}^{\star}\left(f_{0}\right)\right]
$$

For a given configuration, the condition $M \rightarrow \infty$ can be approached as $B_{T} \rightarrow \infty$, and/or as $f_{0} \rightarrow \infty$, as predicted by Weyl's formula. Clearly, these two approaches have limits, as the basic assumption is that of a constant $\partial Q / \partial f$. Other limitations due to the losses are discussed in Section III.

Equation (6) is the cornerstone of the proposed method, since it implies that the same performance that would be obtained only by averaging over a large number of random realizations, can be fairly approximated when using wide-band signals in a single deterministic configuration, provided that the $\mathrm{RC}$ be in an overmoded state. This feature is in particular related to the self-averaging properties of time-reversal, as investigated in [9].

Let us now consider an excitation signal $X_{\mathrm{TR}}(f)$ defined as

$$
X_{\mathrm{TR}}(f)=G(f) \sum_{i=1}^{3} p_{i} \Phi_{i}^{\star}(f, \mathbf{r})=G(f) \Phi^{\mathrm{H}} \mathbf{p}
$$

where $G(f)$ is the spectrum of the pulse to be received at $\mathbf{r}$, while $\mathbf{p}=\left(\begin{array}{lll}p_{1} & p_{2} & p_{3}\end{array}\right)^{\mathrm{T}}$. Applying this to (1) yields a received field

$$
\mathbf{E}_{\mathrm{TR}}=G \boldsymbol{\Phi} \boldsymbol{\Phi}^{\mathrm{H}} \mathbf{p}
$$

having dropped the function arguments for the sake of simplicity. Since we are rather interested in the time-domain field, 


\begin{tabular}{|c|c|c|c|c|c|c|c|c|c|c|c|}
\hline & & & \multicolumn{3}{|c|}{ Rx energy } & \multicolumn{3}{|c|}{ Cross-polarizations } & \multicolumn{3}{|c|}{ 50/90 percentiles $(\mathrm{dB})$} \\
\hline & $f_{0}(\mathrm{GHz})$ & $M_{e}$ & $\mathcal{E}_{1}$ & $\mathcal{E}_{2}$ & $\mathcal{E}_{3}$ & $\rho_{12}$ & $\rho_{13}$ & $\rho_{23}$ & $\left|\rho_{12}\right|$ & $\left|\rho_{13}\right|$ & $\left|\rho_{23}\right|$ \\
\hline \multirow{3}{*}{ Sinc pulse } & 1.0 & 570 & $0.96(0.12)$ & $0.94(0.12)$ & $1.00(0.12)$ & $1.8(5.2)$ & $1.3(4.4)$ & $-2.2(6.3)$ & $28 / 21$ & $30 / 22$ & $26 / 19$ \\
\hline & 1.5 & 420 & $0.97(0.12)$ & $0.94(0.11)$ & $1.00(0.13)$ & $1.2(5.5)$ & $0.5(5.3)$ & $-3.0(6.4)$ & $28 / 20$ & $28 / 22$ & $26 / 18$ \\
\hline & 2.0 & 315 & $0.96(0.11)$ & $0.97(0.11)$ & $1.00(0.12)$ & $1.5(6.8)$ & $1.9(6.3)$ & $-1.2(7.0)$ & $26 / 19$ & $26 / 20$ & $29 / 17$ \\
\hline \multirow{3}{*}{ Gaussian pulse } & 1.0 & & $0.94(0.12)$ & $0.92(0.13)$ & $1.00(0.14)$ & $2.5(5.6)$ & $1.4(4.5)$ & $-2.1(6.8)$ & $28 / 20$ & $29 / 23$ & $26 / 19$ \\
\hline & 1.5 & & $0.96(0.13)$ & $0.94(0.12)$ & $1.00(0.14)$ & $0.3(6.1)$ & $0.4(6.1)$ & $-2.4(7.0)$ & $28 / 19$ & $26 / 21$ & $27 / 17$ \\
\hline & 2.0 & & $0.96(0.11)$ & $0.97(0.12)$ & $1.00(0.14)$ & $1.3(7.4)$ & $1.8(7.6)$ & $-1.2(8.1)$ & $25 / 18$ & $24 / 18$ & $26 / 17$ \\
\hline
\end{tabular}

TABLE I

STATISTICS OF THE PERFORMANCE IN PULSE TRANSMISSION AS OBTAINED FROM THE COLLECTED EXPERIMENTAL DATA. THE FIRST GROUP OF RESULTS DEALS WITH THE ENERGY RECEIVED ALONG THE THREE POLARIZATION, ACCORDING TO THE TRANSFER FUNCTIONS OF THE PROPAGATION CHANNEL. THE SECOND GROUP PRESENTS THE CROSS-POLARIZATION TERMS OF THE POLARIZATION MATRIX $\boldsymbol{\rho}$, WHEREAS IN THE THIRD ONE THE 50

AND 90 PERCENTILES OF THE CROSS-POLARIZATION REJECTION ARE PRESENTED IN DB UNITS. THE AVERAGE VALUES AND THE STANDARD DEVIATIONS ARE GIVEN FOR THE FIRST TWO GROUPS, WITH THE LATTER IN PARENTHESIS. THE CROSS-POLARIZATION TERMS (SECOND GROUP) ARE TO BE DIVIDED BY A FACTOR 100

and especially over the peak of the pulse at $t=0$, we get

$$
\mathbf{e}_{\mathrm{TR}}(0)=\int_{-\infty}^{+\infty} G \boldsymbol{\Phi} \boldsymbol{\Phi}^{\mathrm{H}} \mathbf{p} \mathrm{d} f=\sqrt{\mathcal{E}} \boldsymbol{\rho} \sqrt{\mathcal{E}} \mathbf{p},
$$

having introduced the energy matrix $\mathcal{E}=\operatorname{diag}\left\{\mathcal{E}_{1}, \ldots, \mathcal{E}_{3}\right\}$ with

$$
\mathcal{E}_{i}=\int_{-\infty}^{+\infty} G\left|\Phi_{i}\right|^{2} \mathrm{~d} f=2 \int_{B_{T}} \operatorname{Re}\{G\}\left|\Phi_{i}\right|^{2} \mathrm{~d} f
$$

and the polarization matrix $\rho$, whose elements are defined as

$$
\rho_{i j}=\frac{2 \int_{B_{T}} \operatorname{Re}\left\{G \Phi_{i} \Phi_{j}^{\star}\right\} \mathrm{d} f}{\sqrt{\mathcal{E}_{i} \mathcal{E}_{j}}} .
$$

By applying the ergodic condition (4), it can be proven that

$$
\lim _{M \rightarrow \infty} \boldsymbol{\rho}=\mathrm{E}[\boldsymbol{\rho}]=\mathbf{1}
$$

where $\mathbf{1}$ is the identity matrix. Recalling that in an overmoded RC the field is statistically isotropic, i.e., $\mathrm{E}\left[\left|\Phi_{i}\right|^{2}\right]=$ $\mathrm{E}\left[\left|\Phi_{j}\right|^{2}\right] \forall i, j$, by applying (4) to this last equation too, $\lim _{M \rightarrow \infty} \mathcal{E}_{i}=\mathcal{E}_{0} \forall i$. We can hence claim that

$$
\lim _{M \rightarrow \infty} \mathbf{e}_{\mathrm{TR}}(0)=\mathcal{E}_{0} \mathbf{p}
$$

This result proves that without invoking any statistical averaging process, i.e., no stirring, the pulsed field generated through time-reversal converges, for a sufficiently overmoded RC, to a coherently polarized field, directly controlled by the weight vector $\mathbf{p}$, and this for any static configuration. In other words, the $\Phi_{i}$ functions approximate an orthogonal basis. This result has been derived as an asymptotic property, so that the actual received field is expected to fulfill (13) on average, while presenting a statistical dispersion inversely dependent on $M$.

\section{EXPERIMENTAL RESULTS}

In order to validate the previous analysis, some experimental tests were carried out in SUPELEC's RC, characterized by physical dimensions $3.08 \times 1.84 \times 2.44 \mathrm{~m}^{3}$. The setup consisted of a log-periodic dipole antenna (LPDA), positioned near one corner of the chamber, with the dipoles of the antenna aligned along the vertical direction, i.e., $z$, while the direction of maximum gain was aimed at the corner. Concerning the receiving

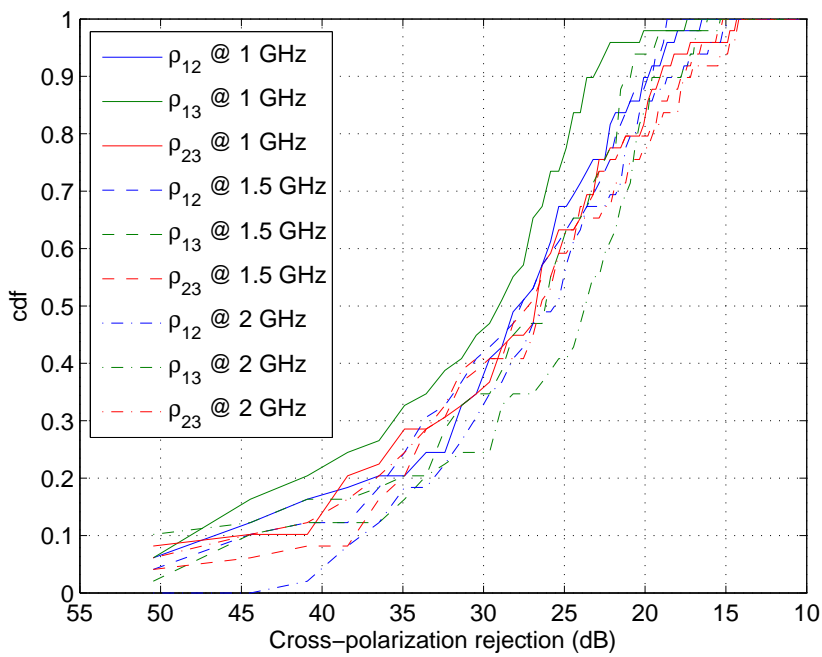

Fig. 2. Empirical cumulative distribution function of the cross-polarization rejections obtained from the experimental results, for a Gaussian pulse. The 50 and 90 percentiles are shown in Table I.

transducer, an all-optical E-field probe was used, manufactured by Enprobe, model EFS-105. This phase-preserving probe is linearly polarized, with a cross-polarization rejection of about $40 \mathrm{~dB}$, thus allowing to test accurately the cross-polarization in the transmitted pulse. The probe was mounted over a styrofoam support, designed in order to ensure the rotation of the probe and thus the measurement of the three Cartesian components of the E field. A total of 50 positions were considered, scattered randomly over the lower half of the RC; for each of these, the transfer functions between the LPDA and the probe was measured along the three polarizations, by means of a vector network analyzer. Three frequencies were considered for $f_{0}$, namely $1,1.5$ and $2 \mathrm{GHz}$, considering a bandwidth of $100 \mathrm{MHz}$ around each frequency. The overmoded condition can be considered as fulfilled for all of these $f_{0}$.

Two types of pulses were considered: 1) a cardinal sine and 2) a Gaussian pulse. The reason for this choice is to check how $\rho$ is affected by a non-flat spectrum. Indeed, the Gaussian pulse, designed to have a $-20 \mathrm{~dB}$ bandwidth of $100 \mathrm{MHz}$, ensured a lower utilization of the ends of its spectrum, and 

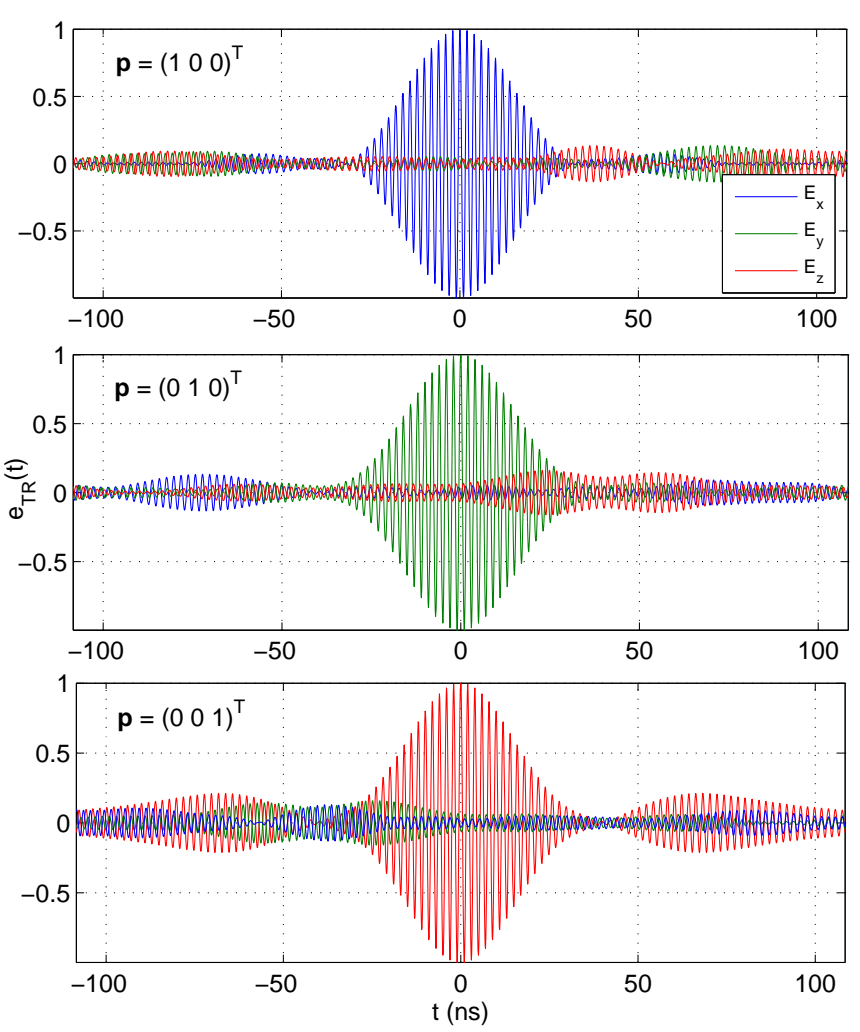

Fig. 3. The field components obtained from experimental results measured at one position, for a Gaussian pulse. Each plot corresponds to a weight vector $\mathbf{p}$ with just one non-null element. Top to bottom, the $x, y$ and $z$ components of the fields are ideally the only excited when the pulse attains its peak value.

thus was expected to have a higher statistical dispersion, since exciting the mods unevenly.

The analysis methodology presented in Section II was applied to the transfer functions, computing, for each frequency and position, the polarization matrix $\rho$, as defined in (11). We first checked the validity of the isotropy assumption, by computing how the energy received along the three polarizations is distributed. The first two statistical moments were computed, and are shown in Table I, showing that this assumption makes sense for the three frequencies we chose, with a maximum error on the average energy of about $8 \%$ and an average one of $5 \%$. Nevertheless, the uncertainty due to randomness of these quantities is of about $20 \%$ across their average value, thus affecting the precision of the copolarization terms.

A similar statistical analysis was carried out on the offdiagonal elements of $\rho$ : the results shown in Table I prove that indeed the field components orthogonal to the originally addressed one are on average very close to zero. The fact that the average is not exactly null is due to positioning errors of the probe: a tilt of its axis of about 1 degree leads to a 0.02 cross-polarization, a value that closely matches the actual averages shown in Table I. The tilt was indeed caused by mechanical errors in the tracks of the styrofoam support housing the probe. The cumulative distribution functions of the cross-polarization projections are shown in Fig. 2, proving that indeed the proposed procedure provides rejections that are much higher than those the LPDA could enable in a free-space environment, at best $20 \mathrm{~dB}$. The median rejection is to be found around $28 \mathrm{~dB}$, and the 90 percentile around $20 \mathrm{~dB}$, while even higher rejections can be found with non negligible probability. In all these results, the Gaussian pulse always underperforms with respect to the sinc one due to a less efficient use of the bandwidth $B_{T}$ and thus a lower $M$.

Concerning the standard deviation of the rejection, it is directly related to the residual error when considering a finite number of modes. Nevertheless, it does not change much when doubling $f_{0}$. This is due to the limited number of degrees of freedom actually available when $Q<\infty$ : it was indeed demonstrated in [10] that of $M$ modes available, a maximum of about $M_{e}=B_{T} Q / f_{0}$ actually participate. This interpretation is supported by the inverse trends followed by the standard deviation and $M_{e}$, as shown in Table I.

\section{CONCLUSIONS}

We have extended the use of time-reversal techniques for generating pulsed fields within reverberation chambers. In particular, we have proven that the polarization of the field can be controlled in a very precise way by simply operating on the signal applied to the excitation antenna. Remarkably, the proposed results are not based on the free-space polarization performance of the excitation antenna, but entirely rely on the decorrelation of the field components inside an RC. Experimental results support this analysis, demonstrating that actual applications can be defined, although further researches are needed in order to be able to predict the statistics of the performance of this technique.

\section{REFERENCES}

[1] D. Hill, "Plane wave integral representation for fields in reverberationchambers," IEEE Transactions on Electromagnetic Compatibility, vol. 40, no. 3, pp. 209-217, 1998.

[2] M. Backstrom and K. Lovstrand, "Susceptibility of electronic systems to high-power microwaves: Summary of test experience," IEEE Transactions on Electromagnetic Compatibility, vol. 46, no. 3, pp. 396-403, 2004.

[3] D. Nitsch, M. Camp, F. Sabath, J. ter Haseborg, and H. Garbe, "Susceptibility of some electronic equipment to HPEM threats," IEEE Transactions on Electromagnetic Compatibility, vol. 46, no. 3, pp. 380389, 2004.

[4] D. Giri, High-Power Electromagnetic Radiators: Nonlethal Weapons and Other Applications. Harvard University Press, 2004.

[5] J. De Rosny, "Milieux réverbérants et réversibilité," Ph.D. dissertation, Université de Paris VI, 2000.

[6] G. Lerosey, J. De Rosny, A. Tourin, A. Derode, G. Montaldo, and M. Fink, "Time reversal of electromagnetic waves," Physical review letters, vol. 92, no. 19, p. 193904, 2004.

[7] H. Moussa, A. Cozza, and M. Cauterman, "Directive wavefronts inside a time reversal electromagnetic chamber," in IEEE EMC Symposium, Austin, Texas, 2009.

[8] A. Shiryaev, Probability. Springer Verlag, New York, 1996.

[9] J. Fouque, J. Garnier, G. Papanicolaou, and K. Solna, Wave propagation and time reversal in randomly layered media. Springer, 2007.

[10] A. Cozza, "Statistics of the performance of time reversal in a lossy reverberating medium," Physical Review E, vol. 80, no. 5, p. 056604 , Nov 2009. 